\title{
Some New Gronwall-Bellman-Type Inequalities on Time Scales and Their Applications
}

\author{
Bin Zheng, ${ }^{1}$ Qinghua Feng, ${ }^{1,2}$ and Fanwei Meng ${ }^{2}$ \\ ${ }^{1}$ School of Science, Shandong University of Technology, Zibo, Shandong 255049, China \\ ${ }^{2}$ School of Mathematical Sciences, Qufu Normal University, Qufu, Shandong 273165, China \\ Correspondence should be addressed to Fanwei Meng; fengqinghua1978@126.com
}

Received 28 May 2013; Accepted 11 October 2013

Academic Editor: Samir H. Saker

Copyright (c) 2013 Bin Zheng et al. This is an open access article distributed under the Creative Commons Attribution License, which permits unrestricted use, distribution, and reproduction in any medium, provided the original work is properly cited.

\begin{abstract}
We establish some new Gronwall-Bellman-type inequalities on time scales. These inequalities are of new forms compared with other Gronwall-Bellman-type inequalities established so far in the literature. Based on them, new bounds for unknown functions are derived. For illustrating the validity of the inequalities established, we present some applications for them, in which the boundedness for solutions for some certain dynamic equations on time scales is researched.
\end{abstract}

\section{Introduction}

As is known, various integral and differential inequalities play an important role in the research of boundedness, global existence, and stability of solutions of differential and integral equations as well as difference equations. Among the investigations for inequalities, generalization of the GronwallBellman-inequality $[1,2]$ is a hot topic, as such inequalities provide explicit bounds for unknown functions concerned. During the past decades, many Gronwall-Bellman-type inequalities have been discovered (e.g., see [3-18]). Recently, with the development of the theory of time scales [19], many integral inequalities on time scales have been established, for example, [20-32], which have proved to be very effective in the analysis of qualitative as well as quantitative analysis of solutions of dynamic equations. But for some certain dynamic equations, for example,

$$
\left(u^{p}(t)\right)^{\Delta}=F\left(t, u(t), u(t), \int_{t}^{\infty} W(\xi, u(\xi)) \Delta \xi\right)
$$

or

$$
u^{p}(t)=C+\int_{t}^{\infty} \widehat{F}\left(s, u(s), u(s), \int_{s}^{\infty} \widehat{W}(\xi, u(\xi)) \Delta \xi\right) \Delta s
$$

$$
+\int_{T}^{\infty} \widehat{F}\left(s, u(s), u(s), \int_{s}^{\infty} \widehat{W}(\xi, u(\xi)) \Delta \xi\right) \Delta s,
$$

it is inadequate to research the boundedness of their solutions by use of the existing results in the literature. So it is necessary to seek new approach to fulfill such analysis for them.

Based on the analysis above, in this paper, we establish some new Gronwall-Bellman-type inequalities on time scales, which are designed so as to be used as a handy tool to research the boundedness of the solutions of the equations mentioned above. By use of the established inequalities, some new bounds for the solutions for the two equations are derived.

In the rest of the paper, $\mathbb{R}$ denotes the set of real numbers, and $\mathbb{R}_{+}=[0, \infty)$. $\mathbb{T}$ denotes an arbitrary time scale, and $\mathbb{T}_{0}=$ $\left[t_{0}, \infty\right) \cap \mathbb{T}$, where $t_{0} \in \mathbb{T}$. On $\mathbb{T}$ we define the forward and backward jump operators $\sigma \in(\mathbb{T}, \mathbb{T})$ and $\rho \in(\mathbb{T}, \mathbb{T})$ such that $\sigma(t)=\inf \{s \in \mathbb{T}, s>t\}$ and $\rho(t)=\sup \{s \in \mathbb{T}, s<t\}$. The graininess $\mu \in\left(\mathbb{T}, \mathbb{R}_{+}\right)$is defined by $\mu(t)=\sigma(t)-t$. Obviously, $\mu(t)=0$ if $\mathbb{T}=\mathbb{R}$, while $\mu(t)=1$ if $\mathbb{T}=\mathbb{Z}$. A point $t \in \mathbb{T}$ with $t>\inf \mathbb{T}$ is said to be left dense if $\rho(t)=t$, right dense if $\sigma(t)=t$, left scattered if $\rho(t)<t$, and right scattered if $\sigma(t)>t$. The set $\mathbb{T}^{\kappa}$ is defined to be $\mathbb{T}$ if $\mathbb{T}$ does not have a left scattered maximum; otherwise it is $\mathbb{T}$ without the left scattered maximum. 
The following definitions and theorems in the theory of time scales are known to us.

Definition 1. A function $f \in(\mathbb{T}, \mathbb{R})$ is called rd-continuous if it is continuous in right-dense points and if the left-sided limits exist in left-dense points, while $f$ is called regressive if $1+\mu(t) f(t) \neq 0 . C_{\mathrm{rd}}$ denotes the set of rd-continuous functions, while $\mathfrak{R}$ denotes the set of all regressive and rdcontinuous functions, and $\mathfrak{R}^{+}=\{f \mid f \in \mathfrak{R}, 1+\mu(t) f(t)>$ $0, \forall t \in \mathbb{T}\}$.

Definition 2. For some $t \in \mathbb{T}^{\kappa}$ and a function $f \in(\mathbb{T}, \mathbb{R})$, the delta derivative of $f$ at $t$ is denoted by $f^{\Delta}(t)$ (provided that it exists) with the property such that, for every $\varepsilon>0$, there exists a neighborhood $\mathfrak{U}$ of $t$ satisfying

$$
\begin{array}{r}
\left|f(\sigma(t))-f(s)-f^{\Delta}(t)(\sigma(t)-s)\right| \leq \varepsilon|\sigma(t)-s| \\
\forall s \in \mathcal{U} .
\end{array}
$$

Definition 3. If $F^{\Delta}(t)=f(t)$ and $t \in \mathbb{T}^{\kappa}$, then $F$ is called an antiderivative of $f$, and the Cauchy integral of $f$ is defined by

$$
\int_{a}^{b} f(t) \Delta t=F(b)-F(a),
$$

where $a, b \in \mathbb{T}^{\kappa}$.

Definition 4. For $p \in \mathfrak{R}$, the exponential function is defined by

$$
e_{p}(t, s)=\exp \left(\int_{s}^{t} \xi_{\mu(\tau)}(p(\tau)) \Delta \tau\right) \quad \text { for } s, t \in \mathbb{T} .
$$

Definition 5. If $\sup _{t \in \mathbb{T}} t=\infty$ and $p \in \Re$, we define

$$
e_{p}(\infty, s)=\exp \left(\int_{s}^{\infty} \xi_{\mu(\tau)}(p(\tau)) \Delta \tau\right) \quad \text { for } t \in \mathbb{T} .
$$

Theorem 6 (see [20, Theorem 2.1]). If $f, g \in(\mathbb{T}, \mathbb{R})$ and $t \in$ $\mathbb{T}^{\mathcal{K}}$, then

(i)

$$
f^{\Delta}(t)= \begin{cases}\frac{f(\sigma(t))-f(t)}{\mu(t)} & \text { if } \mu(t) \neq 0, \\ \lim _{s \rightarrow t} \frac{f(t)-f(s)}{t-s} & \text { if } \mu(t)=0 .\end{cases}
$$

(ii) If $f, g$ are delta differential at $t$, then $f g$ is also delta differential at $t$, and

$$
(f g)^{\Delta}(t)=f^{\Delta}(t) g(t)+f(\sigma(t)) g^{\Delta}(t) .
$$

Theorem 7 (see [20, Theorem 2.2]). If $a, b, c \in \mathbb{T}, \alpha \in \mathbb{R}$, and $f, g \in C_{\mathrm{rd}}$, then one has the following:

(i) $\int_{a}^{b}[f(t)+g(t)] \Delta t=\int_{a}^{b} f(t) \Delta t+\int_{a}^{b} g(t) \Delta t$;

(ii) $\int_{a}^{b}(\alpha f)(t) \Delta t=\alpha \int_{a}^{b} f(t) \Delta t$; (iii) $\int_{a}^{b} f(t) \Delta t=-\int_{b}^{a} f(t) \Delta t$;

(iv) $\int_{a}^{b} f(t) \Delta t=\int_{a}^{c} f(t) \Delta t+\int_{c}^{b} f(t) \Delta t$;

(v) $\int_{a}^{a} f(t) \Delta t=0$;

(vi) if $f(t) \geq 0$ for all $a \leq t \leq b$, then $\int_{a}^{b} f(t) \Delta t \geq 0$.

Theorem 8 (see [29, Theorem 5.2]). If $p \in \mathfrak{R}$, then the following conclusions hold:

(i) $e_{p}(t, t) \equiv 1$ and $e_{0}(t, s) \equiv 1$;

(ii) $e_{p}(\sigma(t), s)=(1+\mu(t) p(t)) e_{p}(t, s)$;

(iii) if $p \in \mathfrak{R}^{+}$, then $e_{p}(t, s)>0$ for all $s, t \in \mathbb{T}$;

(iv) if $p \in \mathfrak{R}^{+}$, then $\ominus p \in \mathfrak{R}^{+}$;

(v) $e_{p}(t, s)=1 /\left(e_{p}(s, t)\right)=e_{\ominus p}(s, t)$,

where $\ominus p=-p /(1+\mu p)$.

Remark 9. If $s=\infty$, then Theorem 8 ((iii), (v)) still holds.

Theorem 10 (see [29, Theorem 5.1]). If $p \in \Re$ and fix $t_{0} \in \mathbb{T}$, then the exponential function $e_{p}\left(t, t_{0}\right)$ is the unique solution of the following initial value problem:

$$
\begin{gathered}
y^{\Delta}(t)=p(t) y(t), \\
y\left(t_{0}\right)=1 .
\end{gathered}
$$

\section{Main Results}

Lemma 11. Suppose that $u, a, m \in C_{\mathrm{rd}}\left(\mathbb{T}_{0}, \mathbb{R}_{+}\right), \widetilde{m}(t)=$ $-m(t) b(t)$, and $\widetilde{m} \in \mathfrak{R}_{+}$. Then

$$
u(t) \leq a(t)+b(t) \int_{t}^{\infty} m(s) u(s) \Delta s, \quad t \in \mathbb{T}_{0},
$$

implies

$$
u(t) \leq a(t)+b(t) \int_{t}^{\infty} e_{\widetilde{m}}(t, \sigma(s)) m(s) a(s) \Delta s, \quad t \in \mathbb{T}_{0} .
$$

Proof. Denote that $v(t)=\int_{t}^{\infty} m(s) u(s) \Delta s$. Then

$$
\begin{gathered}
u(t) \leq a(t)+b(t) v(t), \\
v^{\Delta}(t)=-m(t) u(t) \geq-m(t) b(t) v(t) \\
-m(t) a(t)=\widetilde{m}(t) v(t)-m(t) a(t) .
\end{gathered}
$$

Since $\widetilde{m} \in \mathfrak{R}^{+}$, then from Theorem 8 (iv), we have $\ominus \widetilde{m} \in \mathfrak{R}^{+}$, and furthermore from Theorem 8 (iii), we obtain $e_{\ominus \widetilde{m}}(t, \alpha)>$ 0 for all $\alpha \in \mathbb{T}_{0}$.

According to Theorem 6 (ii),

$$
\left[v(t) e_{\ominus \widetilde{m}}(t, \alpha)\right]^{\Delta}=\left[e_{\ominus \widetilde{m}}(t, \alpha)\right]^{\Delta} v(t)+e_{\ominus \widetilde{m}}(\sigma(t), \alpha) v^{\Delta}(t) .
$$


On the other hand, from Theorem 10, we have

$$
\left[e_{\ominus \widetilde{m}}(t, \alpha)\right]^{\Delta}=(\ominus \widetilde{m})(t) e_{\ominus \widetilde{m}}(t, \alpha) .
$$

So combining (13), (14) and Theorem 8, it follows that

$$
\begin{aligned}
& {\left[v(t) e_{\ominus \widetilde{m}}(t, \alpha)\right]^{\Delta}} \\
& \quad=(\ominus \widetilde{m})(t) e_{\ominus \widetilde{m}}(t, \alpha) v(t)+e_{\ominus \widetilde{m}}(\sigma(t), \alpha) v^{\Delta}(t) \\
& \quad=e_{\ominus \widetilde{m}}(\sigma(t), \alpha)\left[\frac{(\ominus \widetilde{m})(t)}{1+\mu(t)(\ominus \widetilde{m})(t)} v(t)+v^{\Delta}(t)\right] \\
& \quad=e_{\ominus \widetilde{m}}(\sigma(t), \alpha)\left[v^{\Delta}(t)-\widetilde{m}(t) v(t)\right] .
\end{aligned}
$$

Substituting $t$ with $s$, an integration for (15) with respect to $s$ from $\alpha$ to $\infty$ yields

$$
\begin{aligned}
& v(\infty) e_{\ominus \widetilde{m}}(\infty, \alpha)-v(\alpha) e_{\ominus \widetilde{m}}(\alpha, \alpha) \\
& \quad=\int_{\alpha}^{\infty} e_{\ominus \widetilde{m}}(\sigma(s), \alpha)\left[v^{\Delta}(s)-\widetilde{m}(s) v(s)\right] \Delta s .
\end{aligned}
$$

By $v(\infty)=0$ and $e_{\ominus \widetilde{m}}(\alpha, \alpha)=1$, it follows that

$$
\begin{aligned}
& -v(\alpha) \geq-\int_{\alpha}^{\infty} e_{\ominus \widetilde{m}}(\sigma(s), \alpha) m(s) a(s) \Delta s \\
& =-\int_{\alpha}^{\infty} e_{\widetilde{m}}(\alpha, \sigma(s)) m(s) a(s) \Delta s,
\end{aligned}
$$

which is followed by

$$
v(\alpha) \leq \int_{\alpha}^{\infty} e_{\widetilde{m}}(\alpha, \sigma(s)) m(s) a(s) \Delta s .
$$

Since $\alpha \in \mathbb{T}_{0}$ is arbitrary, after substituting $\alpha$ with $t$, we obtain the desired inequality.

Lemma 12. Under the conditions of Lemma 11, furthermore, if $b(t) \equiv 1$ and $a(t)$ is nonincreasing on $\mathbb{T}_{0}$, then

$$
u(t) \leq a(t) e_{-m}(t, \infty), \quad t \in \mathbb{T}_{0} .
$$

Proof. Since $b(t) \equiv 1$ and $a(t)$ is nondecreasing on $\mathbb{T}_{0}$, then $\widetilde{m}=m$ and

$$
\begin{aligned}
u(t) & \leq a(t)+\int_{t}^{\infty} e_{-m}(t, \sigma(s)) a(s) m(s) \Delta s \\
& \leq a(t)\left[1+\int_{t}^{\infty} e_{-m}(t, \sigma(s)) m(s) \Delta s\right] .
\end{aligned}
$$

From [33, Theorems 2.39 and 2.36 (i)], we have

$$
\begin{aligned}
\int_{t}^{\infty} e_{-m}(t, \sigma(s)) m(s) \Delta s \\
\quad=\lim _{x \rightarrow \infty} \int_{x}^{t} e_{-m}(t, \sigma(s))(-m(s)) \Delta s \\
\quad=\lim _{x \rightarrow \infty} e_{-m}(t, x)-e_{-m}(t, t)=e_{-m}(t, \infty)-1 .
\end{aligned}
$$

Combining the above information, we can obtain the desired inequality.
Lemma 13 (see [4]). Assume that $a \geq 0, p \geq q \geq 0$, and $p \neq 0$; then for any $K>0$, the following inequality holds:

$$
a^{q / p} \leq \frac{q}{p} K^{(q-p) / p} a+\frac{p-q}{p} K^{q / p} .
$$

First one will study the following Gronwall-Bellman-type inequality on time scales:

$$
\begin{aligned}
u^{p}(t) \leq & a(t)+b(t) \\
\times & \int_{t}^{\infty}\left[m(s)+f(s) u^{p}(s)\right. \\
& \left.+g(s) u^{q}(s)+\int_{s}^{\infty} h(\xi) u^{r}(\xi) \Delta \xi\right] \Delta s .
\end{aligned}
$$

Theorem 14. Suppose that $u, f, g, h, m, a, b \in C_{\mathrm{rd}}\left(\mathbb{T}_{0}, \mathbb{R}_{+}\right)$, $a, b$ are nonincreasing, $p, q$, and $r$ are constants, $p \geq q \geq$ $0, p \geq r \geq 0$, and $p \neq 0$. If for $t \in \mathbb{T}_{0}, u(t)$ satisfies (23), then

$$
u(t) \leq\left\{\left[a(t)+b(t) H_{2}(t) e_{-H_{3}}(t, \infty)\right] H_{1}(t)\right\}^{1 / p}, \quad t \in \mathbb{T}_{0},
$$

Provided that $\tilde{f},-H_{3} \in \mathfrak{R}_{+}$, where

$$
\begin{aligned}
& H_{1}(t)=1+b(t) \int_{t}^{\infty} e_{\tilde{f}}(t, \sigma(s)) f(s) \Delta s, \\
& \tilde{f}(t)=-f(t) b(t), \\
& H_{2}(t)=\int_{t}^{\infty}\left\{m(s)+g(s)\left[\frac{q}{p} K^{(q-p) / p} a(s)+\frac{p-q}{p} K^{q / p}\right]\right. \\
& \times\left(H_{1}(s)\right)^{q / p} \Delta s \\
& +\int_{t}^{\infty} \int_{s}^{\infty} h(\xi)\left[\frac{r}{p} K^{(r-p) / p} a(\xi)+\frac{p-r}{p} K^{r / p}\right] \\
& \left.\times\left(H_{1}(\xi)\right)^{r / p} \Delta \xi\right\} \Delta s, \quad \forall K>0,
\end{aligned}
$$

$$
\begin{aligned}
H_{3}(t)= & g(t) \frac{q}{p} K^{(q-p) / p} b(t)\left(H_{1}(t)\right)^{q / p} \\
& +\int_{t}^{\infty} h(\xi) \frac{r}{p} K^{(r-p) / p} b(\xi)\left(H_{1}(\xi)\right)^{r / p} \Delta \xi,
\end{aligned}
$$

$$
\forall K>0 \text {. }
$$

Proof. Let the right side of (23) be $v(t)$. Then

$$
\begin{gathered}
u(t) \leq v^{1 / p}(t), \quad t \in \mathbb{T}_{0}, \\
v(t) \leq a(t)+b(t) \\
\times \int_{t}^{\infty}\left[m(s)+f(s) v(s)+g(s) v^{q / p}(s)\right. \\
\left.+\int_{s}^{\infty} h(\xi) v^{r / p}(\xi) \Delta \xi\right] \Delta s .
\end{gathered}
$$


Let

$$
\begin{aligned}
c(t)= & a(t)+b(t) \\
& \times \int_{t}^{\infty}\left[m(s)+g(s) v^{q / p}(s)+\int_{s}^{\infty} h(\xi) v^{r / p}(\xi) \Delta \xi\right] \Delta s .
\end{aligned}
$$

Then we have

$$
v(t) \leq c(t)+b(t) \int_{t}^{\infty} f(s) v(s) \Delta s, \quad t \in \mathbb{T}_{0} .
$$

From Lemma 11, considering $c(t)$ is nonincreasing on $\mathbb{T}_{0}$, we can obtain

$$
\begin{aligned}
v(t) & \leq c(t)+b(t) \int_{t}^{\infty} e_{\tilde{f}}(t, \sigma(s)) c(s) f(s) \Delta s \\
& \leq c(t)\left[1+b(t) \int_{t}^{\infty} e_{\tilde{f}}(t, \sigma(s)) f(s) \Delta s\right] \\
& =c(t) H_{1}(t), \quad t \in \mathbb{T}_{0},
\end{aligned}
$$

where $H_{1}(t), \widetilde{f}(t)$ are defined in (25).

Let

$$
y(t)=\int_{t}^{\infty}\left[m(s)+g(s) v^{q / p}(s)+\int_{s}^{\infty} h(\xi) v^{r / p}(\xi) \Delta \xi\right] \Delta s .
$$

Then

$$
c(t)=a(t)+b(t) y(t) .
$$

From Lemma 13, we have

$$
\begin{aligned}
& (a(t)+b(t) y(t))^{q / p} \\
& \quad \leq \frac{q}{p} K^{(q-p) / p}(a(t)+b(t) y(t))+\frac{p-q}{p} K^{q / p}, \\
& (a(t)+b(t) y(t))^{r / p} \\
& \quad \leq \frac{r}{p} K^{(r-p) / p}(a(t)+b(t) y(t))+\frac{p-r}{p} K^{r / p}, \quad \forall K>0 .
\end{aligned}
$$

A combination of (32), (33), (34), and (35) yields

$$
\begin{aligned}
& y(t) \leq \int_{t}^{\infty}[ m(s)+g(s)\left(c(s) H_{1}(s)\right)^{q / p} \\
&\left.\quad+\int_{t_{0}}^{s} h(\xi)\left(c(\xi) H_{1}(\xi)\right)^{r / p} \Delta \xi\right] \Delta s \\
& \leq \int_{t}^{\infty}\left\{m(s)+g(s)\left[(a(s)+b(s) y(s)) H_{1}(s)\right]^{q / p}\right. \\
&\left.\quad+\int_{s}^{\infty} h(\xi)\left[(a(\xi)+b(\xi) y(\xi)) H_{1}(\xi)\right]^{r / p} \Delta \xi\right\} \Delta s
\end{aligned}
$$

$$
\begin{gathered}
\leq \int_{t}^{\infty}\left\{m(s)+g(s)\left[\frac{q}{p} K^{(q-p) / p}(a(s)+b(s) y(s))\right.\right. \\
\left.\left.+\frac{p-q}{p} K^{q / p}\right]\left(H_{1}(s)\right)^{q / p}\right\} \Delta s \\
+\int_{t}^{\infty} \int_{s}^{\infty} h(\xi)\left[\frac{r}{p} K^{(r-p) / p}(a(\xi)+b(\xi) y(\xi))\right. \\
\left.+\frac{p-r}{p} K^{r / p}\right]\left(H_{1}(\xi)\right)^{r / p} \Delta \xi \Delta s \\
=\int_{t}^{\infty}\left\{m(s)+g(s)\left[\frac{q}{p} K^{(q-p) / p} a(s)+\frac{p-q}{p} K^{q / p}\right]\right. \\
\times\left(H_{1}(s)\right)^{q / p} \\
+\int_{s}^{\infty} h(\xi)\left[\frac{r}{p} K^{(r-p) / p} a(\xi)\right. \\
\left.\left.+\frac{p-r}{p} K^{r / p}\right]\left(H_{1}(\xi)\right)^{r / p} \Delta \xi\right\} \Delta s \\
+\int_{t}^{\infty}\left[g(s) \frac{q}{p} K^{(q-p) / p} b(s)\left(H_{1}(s)\right)^{q / p} y(s)\right. \\
+\int_{s}^{\infty} h(\xi) \frac{r}{p} K^{(r-p) / p} \\
\left.\times b(\xi)\left(H_{1}(\xi)\right)^{r / p} y(\xi) \Delta \xi\right] \Delta s \\
H_{2}(t)+\int_{t}^{\infty} H_{3}(s) y(s) \Delta s,
\end{gathered}
$$

where $H_{2}(t), H_{3}(t)$ are defined in (26) and (27), respectively. By $H_{2}(t)$ being nonincreasing on $\mathbb{T}_{0}$ and $-H_{3} \in \mathfrak{R}_{+}$, according to Lemma 12 , we have

$$
y(t) \leq H_{2}(t) e_{-H_{3}}(t, \infty), \quad t \in \mathbb{T}_{0} .
$$

Combining (32), (34), and (37), we obtain

$$
v(t) \leq\left[a(t)+b(t) H_{2}(t) e_{-H_{3}}(t, \infty)\right] H_{1}(t) .
$$

From (28), (38), we can obtain the desired inequality (13).

Based on Theorem 14, we will establish two VolterraFredholm type delay integral inequalities on time scales in the following two theorems.

Theorem 15. Suppose that $u, f, g, h, m, p, q$, and $r$ are defined as in Theorem 14 with $-f \in \mathfrak{R}_{+}, C>0$ is a constant, 
and $T \in \mathbb{T}_{0}$ is a fixed number. If for $t \in[T, \infty) \cap \mathbb{T}, u(t)$ satisfies the following inequality:

$$
\begin{gathered}
u^{p}(t) \leq C+\int_{t}^{\infty}\left[m(s)+f(s) u^{p}(s)+g(s) u^{q}(s)\right. \\
\left.\quad+\int_{s}^{\infty} h(\xi) u^{r}(\xi) \Delta \xi\right] \Delta s \\
+\int_{T}^{\infty}\left[m(s)+f(s) u^{p}(s)+g(s) u^{q}(s)\right. \\
\left.+\int_{s}^{\infty} h(\xi) u^{r}(\xi) \Delta \xi\right] \Delta s,
\end{gathered}
$$

and furthermore, $\left(1+\widetilde{H}_{21}(T) e_{\widetilde{H}_{3}}(T, \infty)\right) e_{-f}(T, \infty)<2$, then for $t \in[T, \infty) \cap \mathbb{T}$,

$$
\begin{gathered}
u(t) \leq\left\{\left\{\left[\frac{C+\widetilde{H}_{22}(T) e_{-\widetilde{H}_{3}}(T, \infty) e_{f}(T, \infty)}{2-\left(1+\widetilde{H}_{21}(T) e_{-\widetilde{H}_{3}}(T, \infty)\right) e_{f}(T, \infty)}\right]\right.\right. \\
\times\left[1+\widetilde{H}_{21}(t) e_{-\widetilde{H}_{3}}(t, \infty)\right] \\
\left.\left.+\widetilde{H}_{22}(t) e_{-\widetilde{H}_{3}}(t, \infty)\right\} e_{-f}(t, \infty)\right\}^{1 / p},
\end{gathered}
$$

provided that $-\widetilde{H}_{3} \in \mathfrak{R}_{+}$, where

$$
\begin{aligned}
\widetilde{H}_{1}(t)=1+\int_{t}^{\infty} e_{\tilde{f}}(t, \sigma(s)) f(s) \Delta s, \quad \tilde{f}(t)=-f(t) b(t), \\
\widetilde{H}_{21}(t)=\int_{t}^{\infty}\left\{g(s) \frac{q}{p} K^{(q-p) / p}\left(\widetilde{H}_{1}(s)\right)^{q / p}\right. \\
\left.+\int_{s}^{\infty} h(\xi) \frac{r}{p} K^{(r-p) / p}\left(\widetilde{H}_{1}(\xi)\right)^{r / p} \Delta \xi\right\} \Delta s, \\
\forall K>0,
\end{aligned}
$$

$$
\begin{aligned}
\widetilde{H}_{22}(t)=\int_{t}^{\infty}\left\{m(s)+g(s) \frac{p-q}{p} K^{q / p}\left(\widetilde{H}_{1}(s)\right)^{q / p}\right. \\
\left.+\int_{s}^{\infty} h(\xi) \frac{p-r}{p} K^{r / p}\left(\widetilde{H}_{1}(\xi)\right)^{r / p} \Delta \xi\right\} \Delta s,
\end{aligned}
$$$$
\forall K>0 \text {, }
$$

$$
\begin{aligned}
\widetilde{H}_{3}(t)= & g(t) \frac{q}{p} K^{(q-p) / p}\left(\widetilde{H}_{1}(t)\right)^{q / p} \\
& +\int_{t}^{\infty} h(\xi) \frac{r}{p} K^{(r-p) / p}\left(\widetilde{H}_{1}(\xi)\right)^{r / p} \Delta \xi, \quad \forall K>0 .
\end{aligned}
$$

Proof. Let the right side of (39) be $v(t)$. Then

$$
u(t) \leq v^{1 / p}(t), \quad t \in[T, \infty) \bigcap \mathbb{T} .
$$

Considering $v(\infty)=C+\int_{T}^{\infty}\left[m(s)+f(s) u^{p}(s)+g(s) u^{q}(s)+\right.$ $\left.\int_{s}^{\infty} h(\xi) u^{r}(\xi) \Delta \xi\right] \Delta s$, it follows that

$$
\begin{aligned}
v(t)= & v(\infty) \\
& +\int_{t}^{\infty}\left[m(s)+f(s) u^{p}(s)+g(s) u^{q}(s)\right. \\
& \left.\quad+\int_{s}^{\infty} h(\xi) u^{r}(\xi) \Delta \xi\right] \Delta s \\
\leq & v(\infty) \\
& +\int_{t}^{\infty}[m(s)+f(s) v(s) \\
& \left.+g(s) v^{q / p}(s)+\int_{s}^{\infty} h(\xi) v^{r / p}(\xi) \Delta \xi\right] \Delta s, \\
& t \in[T, \infty) \bigcap \mathbb{T} .
\end{aligned}
$$

We notice that the structure of (46) is just similar to that of (29). So following the same manner as the process of (29)-(38) in Theorem 14 (i.e., $v(\infty)$ takes the place of $a(t)$ in Theorem 14, and let $b(t) \equiv 1$ in Theorem 14), considering $\widetilde{H}_{1}(t)=1+\int_{t}^{\infty} e_{-f}(t, \sigma(s)) f(s) \Delta s=e_{-f}(t, \infty)$, we can obtain

$$
\begin{array}{r}
v(t) \leq\left[v(\infty)\left(1+\widetilde{H}_{21}(t) e_{-\widetilde{H}_{3}}(t, \infty)\right)\right. \\
\left.+\widetilde{H}_{22}(t) e_{-\widetilde{H}_{3}}(t, \infty)\right] e_{-f}(t, \infty), \\
t \in[T, \infty) \bigcap \mathbb{T},
\end{array}
$$

where $\widetilde{H}_{21}(t), \widetilde{H}_{22}(t)$, and $\widetilde{H}_{3}(t)$ are defined in (42), (43), and (44), respectively. Setting $t=T$ in (47), we obtain

$$
\begin{aligned}
v(T) \leq[ & v(\infty)\left(1+\widetilde{H}_{21}(T) e_{-\widetilde{H}_{3}}(T, \infty)\right) \\
& \left.+\widetilde{H}_{22}(T) e_{-\widetilde{H}_{3}}(T, \infty)\right] e_{-f}(T, \infty) .
\end{aligned}
$$

As $2 v(\infty)-C=v(T)$, it follows that

$$
\begin{aligned}
& 2 v(\infty)-C=v(T) \\
& \leq\left[v(\infty)\left(1+\widetilde{H}_{21}(T) e_{-\widetilde{H}_{3}}(T, \infty)\right)\right. \\
& \left.+\widetilde{H}_{22}(T) e_{-\widetilde{H}_{3}}(T, \infty)\right] e_{-f}(T, \infty) ;
\end{aligned}
$$

that is,

$$
v(\infty) \leq \frac{C+\widetilde{H}_{22}(T) e_{-\widetilde{H}_{3}}(T, \infty) e_{-f}(T, \infty)}{2-\left(1+\widetilde{H}_{21}(T) e_{-\widetilde{H}_{3}}(T, \infty)\right) e_{-f}(T, \infty)} .
$$

Combining (45), (47), and (50), we can obtain the desired inequality (39).

Theorem 16. Suppose that $u, f, h, p$, and $r$ are defined as in Theorem 14 with $-f \in \mathfrak{R}_{+}, C>0$ is a constant, $T \in \mathbb{T}_{0}$ is a fixed number, $L \in C\left(\mathbb{T}_{0} \times \mathbb{R}_{+}, \mathbb{R}_{+}\right)$, and $0 \leq L(s, x)-L(s, y) \leq$ 
$M(s, y)(x-y)$ for $x \geq y \geq 0$, where $M \in C\left(\mathbb{T}_{0} \times \mathbb{R}_{+}, \mathbb{R}_{+}\right)$. If for $t \in[T, \infty) \cap \mathbb{T}, u(t)$ satisfies the following inequality:

$$
\begin{aligned}
& u^{p}(t) \leq C \\
&+\int_{t}^{\infty}\left[f(s) u^{p}(s)+L(s, u(s))\right. \\
&\left.\quad+\int_{s}^{\infty} h(\xi) u^{r}(\xi) \Delta \xi\right] \Delta s \\
&+\int_{T}^{\infty}\left[f(s) u^{p}(s)+L(s, u(s))\right. \\
&\left.\quad+\int_{s}^{\infty} h(\xi) u^{r}(\xi) \Delta \xi\right] \Delta s,
\end{aligned}
$$

with the initial condition (11), and furthermore, $e_{-\widehat{H}_{2}}(T, \infty) e_{-f}(T, \infty)<2$, then

$$
\begin{aligned}
u(t) \leq\{ & \left\{\left[\frac{C+\widehat{H}_{1}(T) e_{-\widehat{H}_{2}}(T, \infty) e_{-f}(T, \infty)}{2-e_{-\widehat{H}_{2}}(T, \infty) e_{-f}(T, \infty)}+\widehat{H}_{1}(t)\right]\right. \\
& \left.\times e_{-\widehat{H}_{2}}(t, \infty) e_{-f}(t, \infty)\right\}^{1 / p}, \quad t \in[T, \infty) \bigcap \mathbb{T},
\end{aligned}
$$

provided that $-\widehat{H}_{2} \in \Re_{+}$, where

$$
\begin{aligned}
& \widehat{H}_{1}(t)=\int_{t}^{\infty} L\left(s, \frac{p-1}{p} K^{1 / p}\left(e_{-f}(s, \infty)\right)^{1 / p}\right) \Delta s \\
& +\int_{t}^{\infty} \int_{s}^{\infty} h(\xi) \frac{p-r}{p} K^{r / p}\left(e_{-f}(\xi, \infty)\right)^{r / p} \Delta \xi \Delta s, \\
& \widehat{H}_{2}(t)=M\left(t, \frac{p-1}{p} K^{1 / p}\left(e_{-f}(t, \infty)\right)^{1 / p}\right) \\
& \times \frac{1}{p} K^{(1-p) / p}\left(e_{-f}(t, \infty)\right)^{1 / p} \\
& +\int_{t}^{\infty} h(\xi) \frac{r}{p} K^{(r-p) / p}\left(e_{-f}(\xi, \infty)\right)^{r / p} \Delta \xi, \\
& \forall K>0 .
\end{aligned}
$$$$
\forall K>0,
$$

Proof. Let the right side of (51) be $v(t)$. Then

$$
u(t) \leq v^{1 / p}(t), \quad t \in[T, \infty) \bigcap \mathbb{T} .
$$

Furthermore, considering $v(\infty)=C+\int_{T}^{\infty}\left[f(s) u^{p}(s)+\right.$ $\left.L(s, u(s))+\int_{s}^{\infty} h(\xi) u^{r}(\xi) \Delta \xi\right] \Delta s$, we have

$$
\begin{array}{r}
v(t)=v(\infty)+\int_{t}^{\infty}\left[f(s) u^{p}(s)+L(s, u(s))\right. \\
\left.\quad+\int_{s}^{\infty} h(\xi) u^{r}(\xi) \Delta \xi\right] \Delta s \\
\leq v(\infty)+\int_{t}^{\infty}\left[f(s) v(s)+L\left(s, v^{1 / p}(s)\right)\right. \\
\left.+\int_{s}^{\infty} h(\xi) v^{r / p}(\xi) \Delta \xi\right] \Delta s .
\end{array}
$$

Let

$$
c(t)=\int_{t}^{\infty}\left[L\left(s, v^{1 / p}(s)\right)+\int_{s}^{\infty} h(\xi) v^{r / p}(\xi) \Delta \xi\right] \Delta s .
$$

Then

$$
\begin{array}{r}
v(t) \leq v(\infty)+c(t)+\int_{t}^{\infty} f(s) v(s) \Delta s, \\
t \in[T, \infty) \bigcap \mathbb{T} .
\end{array}
$$

Considering $c(t)$ is nonincreasing on $\mathbb{T}_{0}$, by Lemma 12 we obtain

$$
\begin{array}{r}
v(t) \leq(v(\infty)+c(t)) e_{-f}(t, \infty), \\
t \in[T, \infty) \bigcap \mathbb{T} .
\end{array}
$$

Combining (57) and (59), it follows that

$$
\begin{array}{r}
c(t) \leq \int_{t}^{\infty}\left\{L\left(s,\left((v(\infty)+c(s)) e_{-f}(s, \infty)\right)^{1 / p}\right)\right. \\
\left.+\int_{s}^{\infty} h(\xi)\left[(v(\infty)+c(\xi)) e_{-f}(\xi, \infty)\right]^{r / p} \Delta \xi\right\} \Delta s, \\
t \in[T, \infty) \bigcap \mathbb{T} .
\end{array}
$$

On the other hand, from Lemma 13 one can see that the following inequalities hold:

$$
\begin{aligned}
& (v(\infty)+c(t))^{1 / p} \\
& \quad \leq \frac{1}{p} K^{(1-p) / p}(v(\infty)+c(t))+\frac{p-1}{p} K^{1 / p}, \\
& (v(\infty)+c(t))^{r / p} \\
& \quad \leq \frac{r}{p} K^{(r-p) / p}(v(\infty)+c(t))+\frac{p-r}{p} K^{r / p}, \quad \forall K>0 .
\end{aligned}
$$


So combining (60) and (61), we have

$$
\begin{aligned}
& v(\infty)+c(t) \leq v(\infty) \\
& +\int_{t}^{\infty} L\left(s,\left(\frac{1}{p} K^{(1-p) / p}(v(\infty)+c(s))\right.\right. \\
& \left.\left.+\frac{p-1}{p} K^{1 / p}\right)\left(e_{-f}(s, \infty)\right)^{1 / p}\right) \Delta s \\
& +\int_{t}^{\infty} \int_{s}^{\infty} h(\xi)\left[\frac{r}{p} K^{(r-p) / p}(v(\infty)+c(\xi))\right. \\
& \left.+\frac{p-r}{p} K^{r / p}\right] \\
& \times\left(e_{-f}(\xi, \infty)\right)^{r / p} \Delta \xi \Delta s \\
& =v(\infty)+\int_{t}^{\infty}\left\{L \left(s,\left(\frac{1}{p} K^{(1-p) / p}(v(\infty)+c(s))\right.\right.\right. \\
& \left.+\frac{p-1}{p} K^{1 / p}\right) \\
& \left.\times\left(e_{-f}(s, \infty)\right)^{1 / p}\right) \\
& -L\left(s, \frac{p-1}{p} K^{1 / p}\left(e_{-f}(s, \infty)\right)^{1 / p}\right) \\
& \left.+L\left(s, \frac{p-1}{p} K^{1 / p}\left(e_{-f}(s, \infty)\right)^{1 / p}\right)\right\} \Delta s \\
& +\int_{t}^{\infty} \int_{s}^{\infty} h(\xi)\left[\frac{r}{p} K^{(r-p) / p}(v(\infty)+c(\xi))\right. \\
& \left.+\frac{p-r}{p} K^{r / p}\right] \\
& \times\left(e_{-f}(\xi, \infty)\right)^{r / p} \Delta \xi \Delta s \\
& \leq v(\infty)+\int_{t}^{\infty}\left\{M\left(s, \frac{p-1}{p} K^{1 / p}\left(e_{-f}(s, \infty)\right)^{1 / p}\right)\right. \\
& \times\left[\frac{1}{p} K^{(1-p) / p}(v(\infty)+c(s))\right. \\
& \left.\times\left(e_{-f}(s, \infty)\right)^{1 / p}\right] \\
& +L\left(s, \frac{p-1}{p} K^{1 / p}\right. \\
& \left.\left.\times\left(e_{-f}(s, \infty)\right)^{1 / p}\right)\right\} \Delta s \\
& +\int_{t}^{\infty}\left[\int_{s}^{\infty} h(\xi) \frac{r}{p} K^{(r-p) / p}\left(e_{-f}(\xi, \infty)\right)^{r / p} \Delta \xi\right]
\end{aligned}
$$

$$
\begin{aligned}
& \times(v(\infty)+c(s)) \Delta s \\
+ & \int_{t}^{\infty} \int_{s}^{\infty} h(\xi) \frac{p-r}{p} K^{r / p}\left(e_{-f}(\xi, \infty)\right)^{r / p} \Delta \xi \Delta s \\
= & v(\infty)+\widehat{H}_{1}(t)+\int_{t}^{\infty} \widehat{H}_{2}(s)(v(\infty)+c(s)) \Delta s,
\end{aligned}
$$

where $\widehat{H}_{1}(t), \widehat{H}_{2}(t)$ are defined in (53) and (54), respectively.

Considering $\widehat{H}_{1}(t)$ is nonincreasing on $\mathbb{T}_{0}$, then by Lemma 12, we have

$$
\begin{array}{r}
v(\infty)+c(t) \leq\left[v(\infty)+\widehat{H}_{1}(t)\right] e_{-\widehat{H}_{2}}(t, \infty), \\
t \in[T, \infty) \bigcap \mathbb{T} .
\end{array}
$$

Combining (59) and (63), we obtain

$$
\begin{array}{r}
v(t) \leq\left[v(\infty)+\widehat{H}_{1}(t)\right] e_{-\widehat{H}_{2}}(t, \infty) e_{-f}(t, \infty), \\
t \in[T, \infty) \bigcap \mathbb{T} .
\end{array}
$$

Setting $t=T$ in (64), considering $2 v(\infty)-C=v(T)$, we obtain

$$
\begin{aligned}
2 v(\infty)-C & =v(T) \\
& \leq\left[v(\infty)+\widehat{H}_{1}(T)\right] e_{-\widehat{H}_{2}}(T, \infty) e_{-f}(T, \infty),
\end{aligned}
$$

which is followed by

$$
v(\infty) \leq \frac{C+\widehat{H}_{1}(T) e_{-\widehat{H}_{2}}(T, \infty) e_{-f}(T, \infty)}{2-e_{-\widehat{H}_{2}}(T, \infty) e_{-f}(T, \infty)} .
$$

Then combining (55), (64), and (66), we can obtain the desired inequality (52).

\section{Applications}

In this section, we apply the results established above to analysis of boundedness of solutions for certain dynamic equations.

Example 17. Consider the following dynamic equation:

$$
\left(u^{p}(t)\right)^{\Delta}=F\left(t, u(t), u(t), \int_{t}^{\infty} W(\xi, u(\xi)) \Delta \xi\right), \quad t \in \mathbb{T}_{0},
$$

where $u \in C_{\mathrm{rd}}\left(\mathbb{T}_{0}, \mathbb{R}\right), C$ is a constant with $C=u^{p}(\infty)$, and $p>0$ is a constant.

Theorem 18. Suppose that $u(t)$ is a solution of (66)-(67) and assume that $|F(t, x, y, z)| \leq f(t)|x|^{p}+g(t)|y|^{q}+|z|$ and $|W(t, x)| \leq h(t)|x|^{r}$, where $f, g, h, q$, and $r$ are defined the same as in Theorem 14; then the following inequality holds:

$$
|u(t)| \leq\left\{\left[|C|+H_{2}(t) e_{-H_{3}}(t, \infty)\right] H_{1}(t)\right\}^{1 / p}, \quad t \in \mathbb{T}_{0},
$$


where

$$
\begin{aligned}
H_{1}(t)=1+\int_{t}^{\infty} e_{-f}(t, \sigma(s)) f(s) \Delta s, & \begin{aligned}
& H_{2}(t)=\int_{t}^{\infty}\left\{g(s)\left[\frac{q}{p} K^{(q-p) / p}|C|+\frac{p-q}{p} K^{q / p}\right]\right. \\
& \times\left(H_{1}(s)\right)^{q / p} \\
&+\int_{s}^{\infty} h(\xi)\left[\frac{r}{p} K^{(r-p) / p}|C|+\frac{p-r}{p} K^{r / p}\right] \\
&\left.\times\left(H_{1}(\xi)\right)^{r / p} \Delta \xi\right\} \Delta s, \quad \forall K>0, \\
& H_{3}(t)= g(t) \frac{q}{p} K^{(q-p) / p}\left(H_{1}(t)\right)^{q / p} \\
&+\int_{t}^{\infty} h(\xi) \frac{r}{p} K^{(r-p) / p}\left(H_{1}(\xi)\right)^{r / p} \Delta \xi,
\end{aligned} \quad \forall K>0 .
\end{aligned}
$$

Proof. The equivalent integral equation of (56) can be denoted by

$$
u^{p}(t)=C+\int_{t}^{\infty} F\left(s, u(s), u(s), \int_{s}^{\infty} W(\xi, u(\xi)) \Delta \xi\right) \Delta s .
$$

Then we have

$$
\begin{aligned}
& \left|u^{p}(t)\right| \\
& \leq|C|+\int_{t}^{\infty}\left|F\left(s, u(s), u(s), \int_{s}^{\infty} W(\xi, u(\xi)) \Delta \xi\right)\right| \Delta s \\
& \leq|C|+\int_{t}^{\infty}\left[f(s)|u(s)|^{p}+g(s)|u(s)|^{q}\right. \\
& \left.+\left|\int_{s}^{\infty} W(\xi, u(\xi)) \Delta \xi\right|\right] \Delta s \\
& \leq|C|+\int_{t}^{\infty}\left[f(s)|u(s)|^{p}+g(s)|u(s)|^{q}\right. \\
& \left.+\int_{s}^{\infty} h(\xi)|u(\xi)|^{r} \Delta \xi\right] \Delta s .
\end{aligned}
$$

A suitable application of Theorem 14 (i.e., $|C|$ takes the place of $\mathrm{a}(t)$ in Theorem 14, and $b(t) \equiv 1$ in Theorem 14) yields (68).

Remark 19. Under the conditions of Theorem 18, considering $H_{1}(t)=1+\int_{t}^{\infty} e_{-f}(t, \sigma(s)) f(s) \Delta s=e_{-f}(t, \infty)$, furthermore we have the following estimate:

$|u(t)| \leq\left\{\left[|C|+H_{2}(t) e_{-H_{3}}(t, \infty)\right] e_{-f}(t, \infty)\right\}^{1 / p}, \quad t \in \mathbb{T}_{0}$.
Example 20. Consider the following dynamic equation:

$$
\begin{array}{r}
u^{p}(t)=C+\int_{t}^{\infty} \widehat{F}\left(s, u(s), u(s), \int_{s}^{\infty} \widehat{W}(\xi, u(\xi)) \Delta \xi\right) \Delta s \\
+\int_{T}^{\infty} \widehat{F}\left(s, u(s), u(s), \int_{s}^{\infty} \widehat{W}(\xi, u(\xi)) \Delta \xi\right) \Delta s \\
t \in[T, \infty) \bigcap \mathbb{T},
\end{array}
$$

where $u \in C_{\mathrm{rd}}\left(\mathbb{T}_{0}, \mathbb{R}\right), T \in \mathbb{T}_{0}, C$ is a constant with $C=$ $u^{p}(\infty)$, and $p>0$ is a constant.

Theorem 21. Suppose that $u(t)$ is a solution of (73) and assume that $|\widehat{F}(t, x, y, z)| \leq f(t)|x|^{p}+L(t,|y|)+|z|$ and $|\widehat{W}(t, x)| \leq h(t)|x|^{r}$, where $f, h, r$, and L are defined the same as in Theorem 16. Then the following inequality holds:

$$
\begin{aligned}
u(t) \leq & {\left[\frac{|C|+\widehat{H}_{1}(T) e_{-\widehat{H}_{2}}(T, \infty) e_{-f}(T, \infty)}{2-e_{-\widehat{H}_{2}}(T, \infty) e_{-f}(T, \infty)}+\widehat{H}_{1}(t)\right] } \\
& \times e_{-\widehat{H}_{2}}(t, \infty) e_{-f}(t, \infty), \quad t \in[T, \infty] \bigcap \mathbb{T},
\end{aligned}
$$

provided that $e_{-\widehat{H}_{2}}(T, \infty) e_{-f}(T, \infty)<2$ and $-f,-\widehat{H}_{2} \in \mathfrak{R}^{+}$, where $\widehat{H}_{1}(t), \widehat{H}_{2}(t)$ are defined as in Theorem 16.

Proof. From (73), we have

$$
\begin{aligned}
&\left|u^{p}(t)\right| \leq|C|+\int_{t}^{\infty}\left|\widehat{F}\left(s, u(s), u(s), \int_{\infty}^{s} \widehat{W}(\xi, u(\xi)) \Delta \xi\right)\right| \Delta s \\
&+\int_{T}^{\infty}\left|\widehat{F}\left(s, u(s), u(s), \int_{\infty}^{s} \widehat{W}(\xi, u(\xi)) \Delta \xi\right)\right| \Delta s \\
& \leq|C|+\int_{t}^{\infty}\left[f(s)|u(s)|^{p}+L(s,|u(s)|)\right. \\
&+\int_{T}^{\infty}\left[f(s)\left|\int_{\infty}^{s} \widehat{W}(\xi, u(\xi)) \Delta \xi\right|\right] \Delta s \\
&\left.+\left|\int_{\infty}^{s} \widehat{W}(\xi, u(\xi)) \Delta \xi\right|\right] \Delta s \\
& \leq|C|+\int_{t}^{\infty}\left[f(s)|u(s)|^{p}+L(s,|u(s)|)\right. \\
&+\int_{T}^{\infty}\left[f(s)|u(s)|^{p}+L(s,|u(s)|)\right. \\
&\left.+\int_{\infty}^{s} h(\xi)|u(\xi)|^{r}\right] \Delta s . \\
&\left.+\int_{\infty}^{s} h(\xi)|u(\xi)|^{r}\right] \Delta s
\end{aligned}
$$

Then under the condition $e_{-\widehat{H}_{2}}(T, \infty) e_{-f}(T, \infty)<2$, a suitable application of Theorem 16 yields (74). 


\section{Acknowledgments}

This work was partially supported by Natural Science Foundation of Shandong Province (China) (Grant no. ZR2013AQ009) and Doctoral initializing Foundation of Shandong University of Technology (China) (Grant no. 4041413030).

\section{References}

[1] T. H. Gronwall, "Note on the derivatives with respect to a parameter of the solutions of a system of differential equations," Annals of Mathematics, vol. 20, no. 4, pp. 292-296, 1919.

[2] R. Bellman, "The stability of solutions of linear differential equations,” Duke Mathematical Journal, vol. 10, pp. 643-647, 1943.

[3] Ou Yang-Liang, "The boundedness of solutions of linear differential equations $y^{\prime \prime}+A(t) y=0$," Advances in Mathematics, vol. 3, pp. 409-415, 1957.

[4] F. Jiang and F. Meng, "Explicit bounds on some new nonlinear integral inequalities with delay," Journal of Computational and Applied Mathematics, vol. 205, no. 1, pp. 479-486, 2007.

[5] A. Gallo and A. M. Piccirillo, "About some new generalizations of Bellman-Bihari results for integro-functional inequalities with discontinuous functions and applications," Nonlinear Analysis A, vol. 71, no. 12, pp. e2276-e2287, 2009.

[6] W. S. Cheung and J. Ren, "Discrete non-linear inequalities and applications to boundary value problems," Journal of Mathematical Analysis and Applications, vol. 319, no. 2, pp. 708-724, 2006.

[7] W. N. Li, M. Han, and F. W. Meng, "Some new delay integral inequalities and their applications," Journal of Computational and Applied Mathematics, vol. 180, no. 1, pp. 191-200, 2005.

[8] O. Lipovan, "A retarded integral inequality and its applications," Journal of Mathematical Analysis and Applications, vol. 285, no. 2, pp. 436-443, 2003.

[9] Q. H. Ma and E. H. Yang, "Some new Gronwall-Bellman-Bihari type integral inequalities with delay," Periodica Mathematica Hungarica, vol. 44, no. 2, pp. 225-238, 2002.

[10] W. S. Wang, "Some generalized nonlinear retarded integral inequalities with applications," Journal of Inequalities and Applications, vol. 2012, article 31, 14 pages, 2012.

[11] Q. H. Ma, "Estimates on some power nonlinear VolterraFredholm type discrete inequalities and their applications," Journal of Computational and Applied Mathematics, vol. 233, no. 9, pp. 2170-2180, 2010.

[12] L. Li, F. Meng, and L. He, "Some generalized integral inequalities and their applications," Journal of Mathematical Analysis and Applications, vol. 372, no. 1, pp. 339-349, 2010.

[13] O. Lipovan, "Integral inequalities for retarded Volterra equations," Journal of Mathematical Analysis and Applications, vol. 322, no. 1, pp. 349-358, 2006.

[14] B. G. Pachpatte, "Explicit bounds on certain integral inequalities," Journal of Mathematical Analysis and Applications, vol. 267, no. 1, pp. 48-61, 2002.

[15] W. S. Wang, "A class of retarded nonlinear integral inequalities and its application in nonlinear differential-integral equation," Journal of Inequalities and Applications, vol. 2012, article 154, 10 pages, 2012.

[16] W. S. Wang, "Some retarded nonlinear integral inequalities and their applications in retarded differential equations," Journal of Inequalities and Applications, vol. 2012, article 75, 8 pages, 2012.
[17] R. A. C. Ferreira and D. F. M. Torres, "Generalized retarded integral inequalities," Applied Mathematics Letters, vol. 22, no. 6, pp. 876-881, 2009.

[18] R. Xu and Y. G. Sun, "On retarded integral inequalities in two independent variables and their applications," Applied Mathematics and Computation, vol. 182, no. 2, pp. 1260-1266, 2006.

[19] S. Hilger, "Analysis on measure chains-a unified approach to continuous and discrete calculus," Results in Mathematics, vol. 18 , no. $1-2$, pp. $18-56,1990$.

[20] W. N. Li, "Some new dynamic inequalities on time scales," Journal of Mathematical Analysis and Applications, vol. 319, no. 2, pp. 802-814, 2006.

[21] F. H. Wong, C. C. Yeh, and W. C. Lian, “An extension of Jensen's inequality on time scales," Advances in Dynamical Systems and Applications, vol. 1, no. 1, pp. 113-120, 2006.

[22] M. Z. Sarikaya, "On weighted Iyengar type inequalities on time scales," Applied Mathematics Letters, vol. 22, no. 9, pp. 1340$1344,2009$.

[23] Q. Feng and B. Zheng, "Generalized Gronwall-Bellman-type delay dynamic inequalities on time scales and their applications," Applied Mathematics and Computation, vol. 218, no. 15, pp. 7880-7892, 2012.

[24] S. H. Saker, "Some nonlinear dynamic inequalities on time scales," Mathematical Inequalities \& Applications, vol. 14, no. 3, pp. 633-645, 2011.

[25] M. Bohner and T. Matthews, "The Grüss inequality on time scales," Communications in Mathematical Analysis, vol. 3, no. 1, pp. 1-8, 2007.

[26] Q. A. Ngô, "Some mean value theorems for integrals on time scales," Applied Mathematics and Computation, vol. 213, no. 2, pp. 322-328, 2009.

[27] W. Liu and Q. A. Ngô, "Some Iyengar-type inequalities on time scales for functions whose second derivatives are bounded," Applied Mathematics and Computation, vol. 216, no. 11, pp. 3244-3251, 2010

[28] S. H. Saker, "Some nonlinear dynamic inequalities on time scales and applications," Journal of Mathematical Inequalities, vol. 4, pp. 561-579, 2010.

[29] R. Agarwal, M. Bohner, and A. Peterson, "Inequalities on time scales: a survey," Mathematical Inequalities and Applications, vol. 4, no. 4, pp. 535-557, 2001.

[30] W. S. Cheung and Q. H. Ma, "On certain new GronwallOu-Iang type integral inequalities in two variables and their applications," Journal of Inequalities and Applications, vol. 2005, no. 4, pp. 347-361, 2005.

[31] C. J. Chen, W. S. Cheung, and D. Zhao, "Gronwall-bellmantype integral inequalities and applications to BVPs," Journal of Inequalities and Applications, vol. 2009, Article ID 258569, 15 pages, 2009.

[32] O. Lipovan, "A retarded Gronwall-like inequality and its applications," Journal of Mathematical Analysis and Applications, vol. 252, no. 1, pp. 389-401, 2000.

[33] M. Bohner and A. Peterson, Dynamic Equations on Time Scales: An Introduction with Applications, Birkhäuser, Boston, Mass, USA, 2001. 


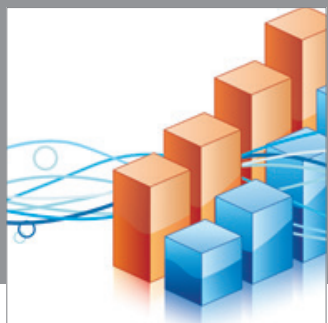

Advances in

Operations Research

mansans

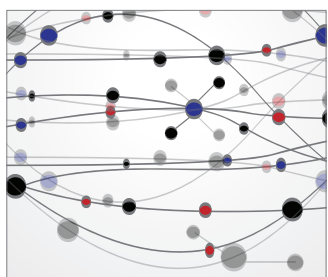

The Scientific World Journal
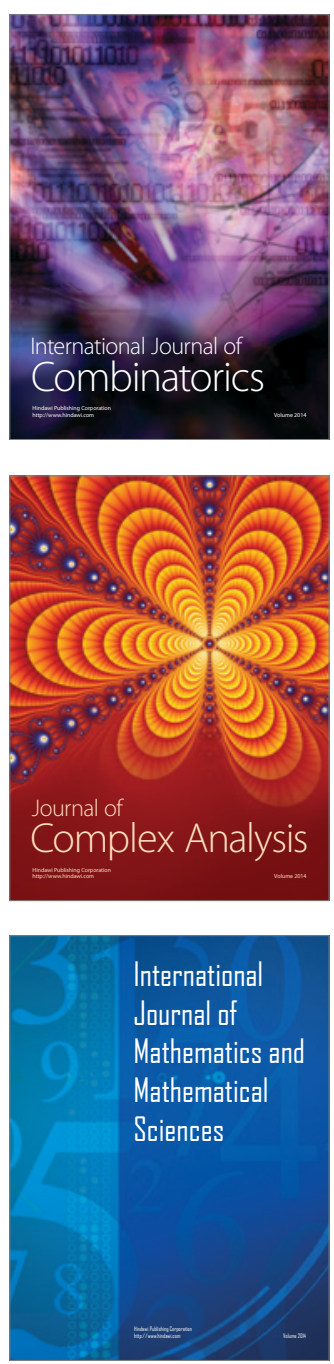
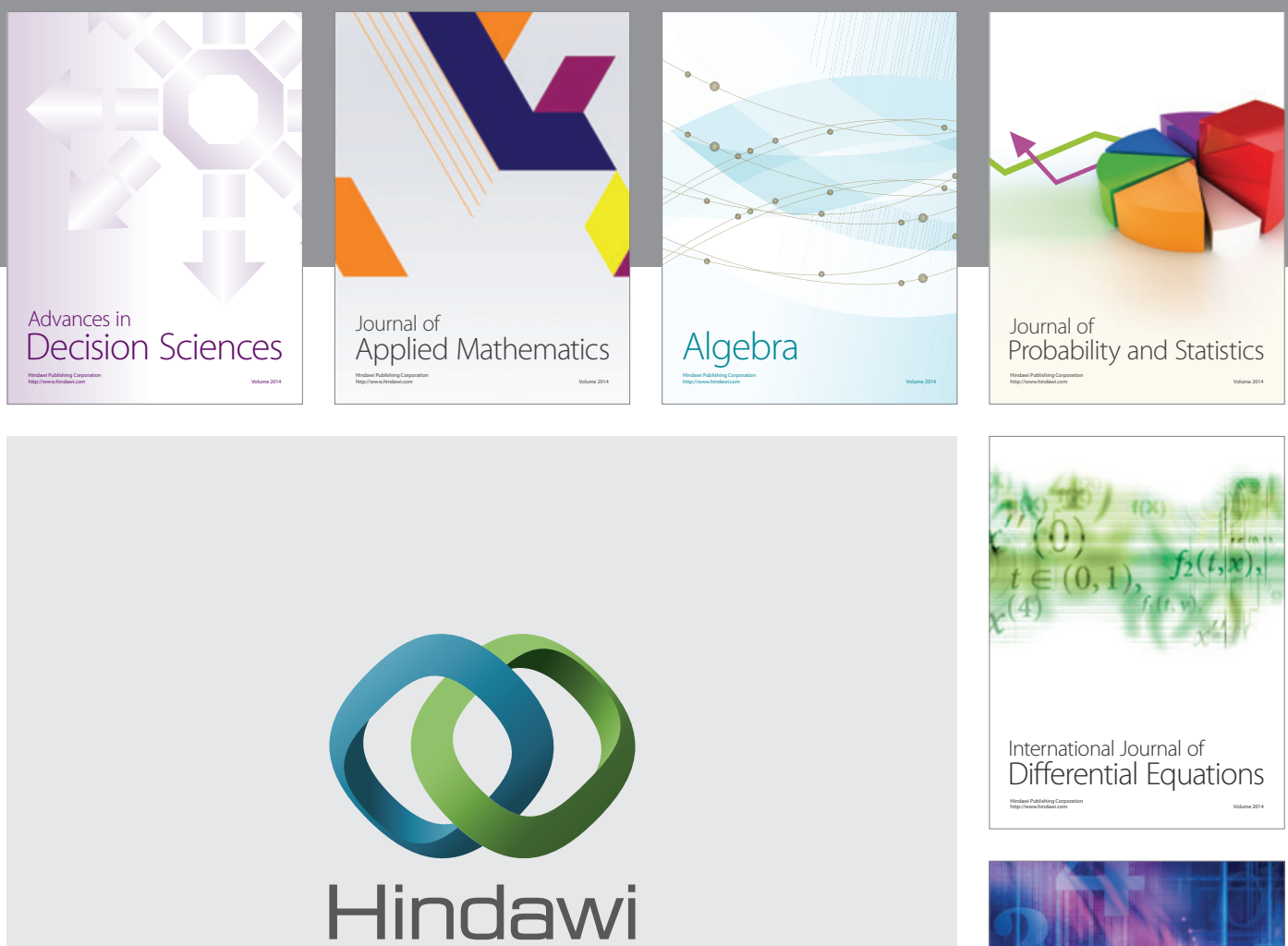

Submit your manuscripts at http://www.hindawi.com
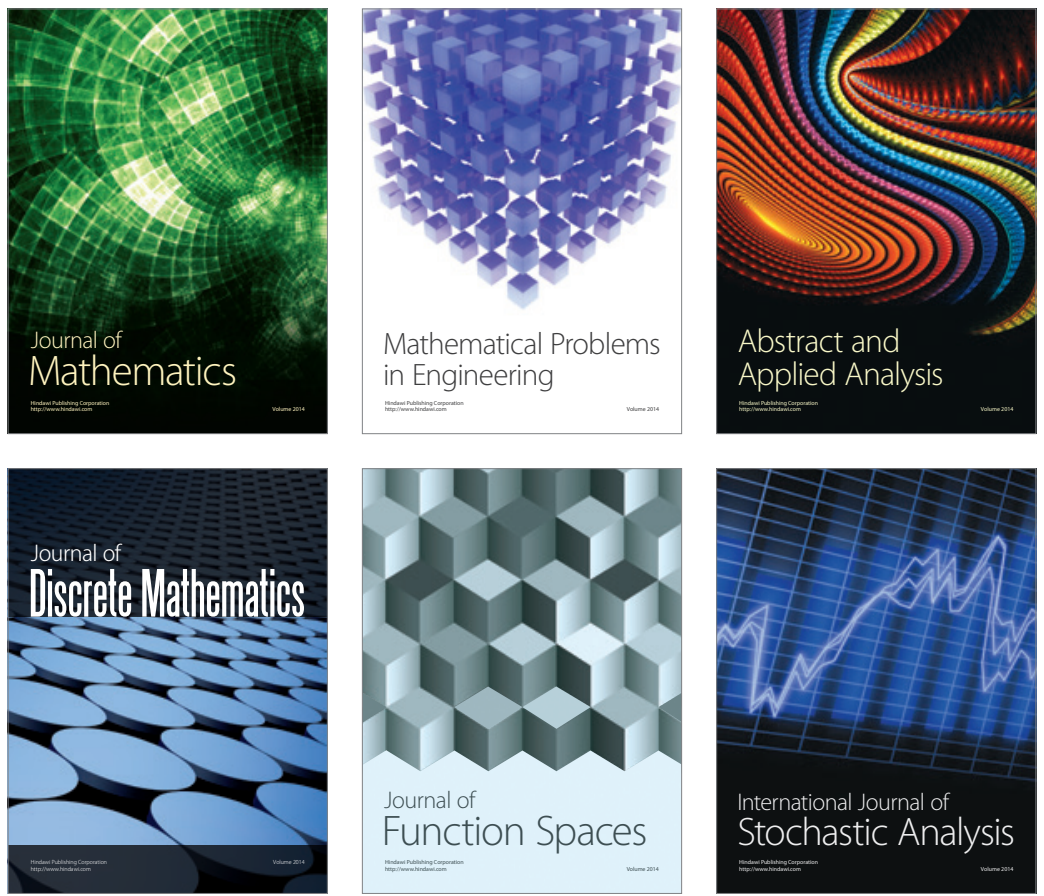

Journal of

Function Spaces

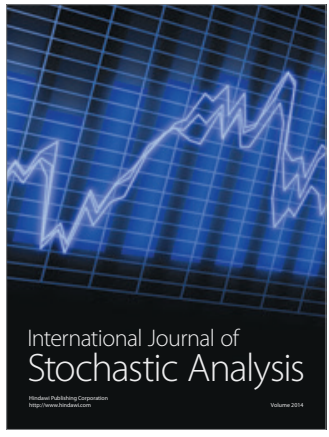

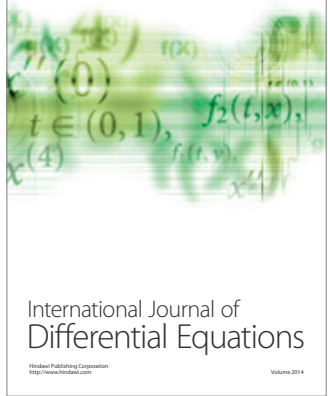
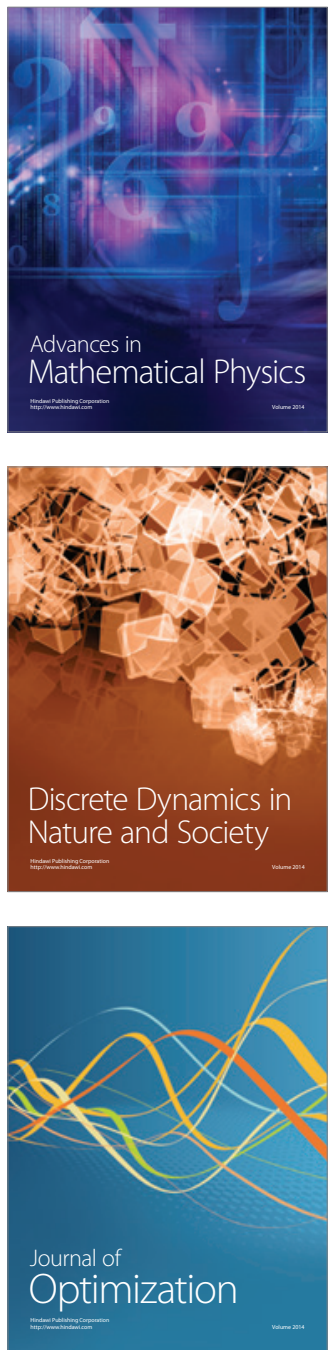